\title{
Do AGN suppress star formation in disc-dominated galaxies?
}

\author{
B. Husemann ${ }^{1}$, J. Walcher ${ }^{1}$, L. Wisotzki ${ }^{1}$, J. Gerssen $^{1}$, K. Jahnke ${ }^{2}$, \\ S. F. Sánchez ${ }^{3}$, and V. Wild ${ }^{4}$ \\ ${ }^{1}$ Leibniz-Institut für Astrophysik Potsdam, Germany \\ email: bhusemann@aip.de \\ ${ }^{2}$ Max-Planck-Institut für Astronomie, Heidelberg, Germany \\ ${ }^{3}$ Instituto de Astrofísica de Andalucía (CSIC), Granada, Spain \\ ${ }^{4}$ University of St. Andrews, United Kingdom
}

\begin{abstract}
We present preliminary results of our integral field spectroscopy (IFS) observations to test whether AGN can suppress star formation in disc-dominated galaxies. We find a lower specific star formation rate and a different radial $\mathrm{H} \alpha$ profile for AGN than in the control sample.
\end{abstract}

Keywords. galaxies: active - HII regions - galaxies: spiral

We selected a sample of 20 nearby AGN and 20 normal star-forming disc galaxies for IFS follow-up to obtain spatially resolved emission-line maps of optical lines. The galaxies cover a narrow redshift and stellar mass range, paying close attention to matching galaxy properties. We use classical emission-line diagnostics to identify HII regions across the galaxies. Assuming that the $\mathrm{H} \alpha$ emission from HII regions is a proxy for ongoing star formation, we find (i) the integrated specific star formation rate (sSFR) to be systematically lower by $0.2 \mathrm{dex}$ (Fig. 1) in AGN hosts, and (ii) the radial $\mathrm{H} \alpha$ surface luminosity to have a larger dispersion closer to the nucleus in AGN hosts than in the control sample. We interpret this as tentative evidence for the suppression of star formation in AGN host galaxies. Better statistics will be provided soon by the ongoing CALIFA survey (Sánchez et al. 2012) for a much larger sample of galaxies that will strengthen the results.

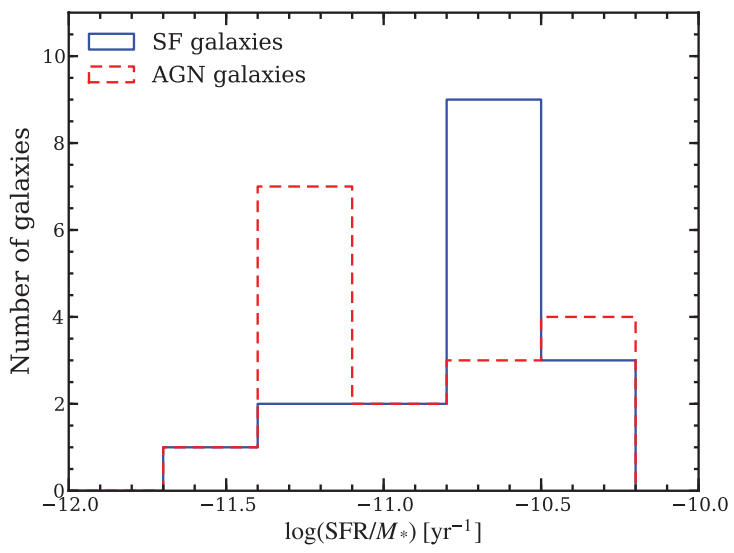

Figure 1. Distribution of the total sSFR for our star forming and AGN host galaxies.

\section{Reference}

Sánchez et al. 2012, A\&̈A, 538, A8 